\title{
A PRESENÇA DE HENRI LEFEBVRE NO DEBATE ACADÊMICO CONTEMPORÂNEO DA ARQUITETURA
}

\section{THE PRESENCE OF HENRI LEFEBVRE IN THE CONTEMPORARY ACADEMIC DEBATE OF ARCHITECTURE}

\author{
Leandro Silva Medrano 1 \\ Faculdade de Arquitetura e Urbanismo, Universidade de São Paulo, São Paulo, SP, Brasil, medrano@ usp.br \\ Luiz Antonio Recamán Barros ${ }^{2}$ \\ Faculdade de Arquitetura e Urbanismo, Universidade de São Paulo, São Paulo, SP, Brasil, recaman@usp.br \\ Mariana Martinez Wilderom Chagas ${ }^{3}$ \\ Programa de Pós-Graduação em Arquitetura e Urbanismo, Universidade de São Paulo, São Paulo, SP, Brasil, \\ mariana.wilderom@usp.br
}

Raphael Grazziano 4

Programa de Pós-Graduação em Arquitetura e Urbanismo, Universidade de São Paulo, São Paulo, SP, Brasil, raphael.grazziano@gmail.com

\section{Resumo}

A grande produção de Henri Lefebvre (HL) sobre cidades, espaços urbanos, espaço urbano e sociedade poderia indicar sua relevância para a arquitetura produzida após o auge do ciclo neoliberal e sua crise em 2008. Este autor introduziu a base prático-sensível na dialética entre os processos espacial e social para discutir as dimensões ideológicas que fundamentam a intervenção urbanística e arquitetônica levada a cabo pela racionalidade do Estado indicar sua relevância à arquitetura produzida após o apogeu do ciclo neoliberal e a sua crise em 2008. O objetivo deste artigo é identificar a relação entre os conceitos de HL e a arquitetura no debate acadêmico contemporâneo, no qual a cidade e o urbano são destacados. Como metodologia utilizamos a Revisão Sistemática de Literatura (RSL), com critérios de filtragem que buscavam a relação entre a obra de HL, destacada em revistas especializadas internacionais indexadas, sobre o espaço e a cidade e as preocupações mais diretamente voltadas para a arquitetura e desenho urbano. Conclui-se que a influência da obra Lefebvriana nos temas arquitetônicos é pouco expressiva no âmbito acadêmico detectado aqui, e isso é um problema, se considerarmos as possibilidades compreensivas e críticas dessa obra, principalmente sob as determinações da globalização e a mais recente apropriação imediatamente política do "direito à cidade" (DC).

Palavras-chave: Henri Lefebvre. Arquitetura. Desenho urbano.

\begin{abstract}
Keywords: Henri Lefebvre. Architecture. Urban design. Henri Lefebvre's (HL) large production about cities, urban, urban space and society could indicate its relevance to the architecture produced after the heyday of the neoliberal cycle and its crisis in 2008. This author introduced the practical-sensitive basis in the debate between the spatial and social processes to discuss the ideological dimensions that underpin the urban and architectural intervention carried out by the rationality of the State. This paper aims to identify the relationship between HL concepts and architecture in the contemporary academic debate, in which the city and the urban are highlighted. The Systematic Literature Review method was used as well as a refining criterion that looked for the relation between the work of HL, selected in specialized indexed international journals, on the space and the city and the concerns more directly related to the architecture and urban design. Concluding, we point out that the influence of Lefebvrian work on architectural subjects is not substantial in the academic scope here detected. This is understood a problem if we consider the comprehensive and critical possibilities of this work, especially under the determinations of globalization and the most recent immediate political appropriation of the "right to the city".
\end{abstract}

Keywords: Henri Lefebvre. Architecture. Urban design.

How to cite this article:

MEDRANO, Leandro Silva et al. A presença de Henri Lefebvre no debate acadêmico contemporâneo da arquitetura. PARC Pesquisa em Arquitetura e Construção, Campinas, SP, v. 8, n. 3, p. 170-180, set. 2017. ISSN 1980-6809. Disponível em: $<$ <ttps://periodicos.sbu.unicamp.br/ojs/index.php/parc/article/view/8650261>. Acesso em: 20 jan. 2018. doi:http://dx.doi.org/10.20396/parc.v8i3.8650261. 


\section{Introdução}

Este trabalho responde à edição especial da Revista PARC dedicada a Revisões Sistemáticas da Literatura (RSL) (cf. literatura proposta pela revista: COOPER; HEDGES, 2009; DRESCH; LACERDA; ANTUNES JR., 2015; GOUGH; THOMAS, 2012). Nesse sentido, entendemos que as conclusões aqui apresentadas referem-se exclusivamente ao método proposto. Esse trabalho é um exercício de aproximação aos temas subjacentes à obra de Henri Lefebvre (HL), e faz parte de uma abordagem mais ampla sobre sua obra.

O Grupo de Pesquisa [oculto para anonimato] se organizou em torno de questões que perscrutavam a relação entre arquitetura e sociedade, com um horizonte que procura compreender os limites da disciplina no processo de formação da arquitetura brasileira. Mais especificamente, buscar os padrões espaciais inerentes a essa tradição constituída durante o século XX e sua relação conflituosa com os problemas sociais e urbanos a que, a princípio, fora autorizada a transformar. Nesse sentido, temas como "forma urbana", "forma arquitetônica" e "espaço social" se tornaram os eixos por meio dos quais se poderia iniciar uma reflexão crítica. A intenção, de modo abrangente, é fazer incidir a crítica - bastante consolidada no debate urbano e social brasileiro - nos meios que a disciplina arquitetônica dispõe para sua atuação e intervenção.

Essa perspectiva confluiu necessariamente para a vasta produção de HL sobre a cidade, o urbano, o espaço e a sociedade urbana. Esse autor introduziu a base práticosensível na dialética entre o processo espacial e o processo social. Sua tríade espacial - o vivido, o concebido e o percebido - do mesmo modo, se mostra essencial para discutir as dimensões ideológicas que fundamentam a intervenção urbanística e arquitetônica levadas a cabo pela racionalidade do Estado. Essa dimensão planejada do espaço tensiona-se, sem se confundir, com as dimensões espaciais simbólicas e (re)produtivas das forças econômicas. $\mathrm{O}$ interesse dessas formulações sobre a cidade e o espaço para a arquitetura tem sido recentemente destacado (LEFEBVRE, 2014; STANEK, 2011; STANEK; SCHMID; MORAVÁNSZKY, 2014).

Além disso, após a crise urbano-financeira de 2008, o tema das cidades e do ativismo urbano ressurge com força (HARVEY, 2012), depois de décadas de anúncio do fim do urbano (KOOLHAAS; MAU, 1995). Ao mesmo tempo que a crise ideológica do neoliberalismo coloca as cidades como o centro do debate contemporâneo, a arquiteturaespetáculo que lhe dava expressão encontra o seu limite ideológico inevitável e já criticamente previsto por diferentes autores (e.g. ARANTES, 1998; JAMESON, 2001). Assim, os caminhos da cidade e da arquitetura parecem se cruzar novamente.
Nesse sentido propôs-se uma leitura sistemática sobre o estado atual da recepção do debate lefebvriano concernente aos temas acima destacados. Os critérios de busca adotados procuraram captar a produção pós-2008 nos periódicos especializados da área que tratassem de temas da obra de HL relacionados a arquitetura.

O objetivo deste trabalho é, portanto, detectar a presença da obra de HL nos debates acadêmicos atuais da arquitetura por meio da incidência das referências a esse autor. Além disso, objetivamos precisar quais aportes teóricos desse autor são mais influentes nesse contexto por exemplo, o Direito à Cidade (DC), a tríade espacial e a vida cotidiana.

O artigo se divide em quatro partes: 1) Metodologia: trata dos parâmetros adotados para a aplicação do método RSL; 2) Métricas: análise quantitativa dos dados levantados nos artigos da amostra; 3) Discussão dos resultados: análise dos dados considerando o enfoque proposto que relaciona: arquitetura, urbanismo e sociedade, principalmente quando referentes ao Brasil e à questão do subdesenvolvimento; 4) Considerações finais.

\section{Metodologia RSL}

A metodologia RSL traz a vantagem de abordar o recorte disciplinar de modo amplo e sistemático, principalmente considerando nosso interesse em compreender a recepção de HL no campo da arquitetura. Contudo, a obra de HL requer uma análise prioritariamente qualitativa e crítica, o que demandou um recorte do universo de referências trabalhadas. Para tanto, o levantamento bibliográfico restringiu-se a artigos científicos publicados entre 2008 e 2017 - cobrindo uma década de publicações desde o marco inicial da crise urbano-financeira. Os artigos foram procurados em três bases de dados: Web of Science (Principal Coleção), Scopus e Scielo, em consulta realizada entre maio e junho de 2017. Para compreender como a obra de HL é lida na mediação entre arquitetura, urbanismo e sociedade, foram escolhidas as seguintes entradas de busca: (architect* AND Lefebvre), (urbanis* AND Lefebvre), (design* AND Lefebvre) e ("right to the city" AND Lefebvre), submetidas nos campos Topic (Web of Science), "Title, abstract, keywords" (Scopus) e "All indexes" (Scielo).

O conjunto das buscas revelou 469 resultados. Esse número foi reduzido para 275, eliminando os resultados duplicados nas diferentes buscas.

Com o objetivo de alcançar os artigos de maior relevância de acordo com critérios usuais de avaliação, foram descartados todos os artigos em periódicos com avaliação menor do que A segundo o Qualis CAPES (dentro da área de arquitetura, caso o periódico tenha sido nela avaliado; caso contrário, na disciplina mais bem avaliada 
disponível). Caso o periódico não possuísse nenhuma avaliação no Qualis, foram então considerados apenas os periódicos com índice de impacto igual ou superior a 0.5 , ou presentes no portal da Scielo, pois ambos são critérios para uma avaliação A. A partir desse filtro, restaram 110 artigos.

Em seguida, cinco foram descartados por não se tratarem efetivamente de artigos científicos, tendo passado pelos filtros anteriores; três estavam indisponíveis para consulta; e um foi eliminado por estar em língua desconhecida por todos os integrantes da pesquisa.

Para produzir um material que enfocasse mais diretamente o conjunto de preocupações da pesquisa - em suma, os aportes lefebvrianos mais diretamente ligados a questões de arquitetura e desenho urbano - foi realizada uma sequência de triagens, por leitura de títulos, resumos e, em casos menos claros, introduções e conclusões (cf. método em DRESCH; LACERDA; ANTUNES JR., 2015, p. 143). 21 artigos foram descartados por não pertencerem diretamente à área de arquitetura e urbanismo; outros 15 foram eliminados ou por não tratarem de temas especificamente relacionados a arquitetura e urbanismo, que é questão central desta revisão de literatura, ou por usarem a bibliografia lefebvriana de modo apenas pontual.

Restaram, portanto, 65 artigos aptos para a leitura analítica de seu conteúdo completo (DRESCH; LACERDA; ANTUNES JR., 2015, p. 144) e que foram considerados para as distribuições estatísticas do item 2 deste artigo.

\section{Métricas}

Um conjunto de gráficos foi elaborado para a análise quantitativa da amostra de artigos.

O Gráfico 1 revela a distribuição geográfica dos artigos selecionados, segundo a afiliação institucional do autor principal. Note-se que há forte predominância de literatura anglo-saxã no conjunto (60\%, considerados Austrália, Canadá, Escócia, EUA, Inglaterra, Irlanda do Norte e País de Gales), provavelmente resultante não só de maior produção acadêmica nesses países, mas também de maior quantidade de periódicos em inglês no Qualis A e nas bases de dados consultadas. Afiliações de países subdesenvolvidos são minoria na amostra (13\%, considerados Argentina, Brasil, China, Índia, Qatar, Turquia) (Gráfico 2).

Gráfico 2 - Afiliação institucional do autor principal, por categoria de desenvolvimento

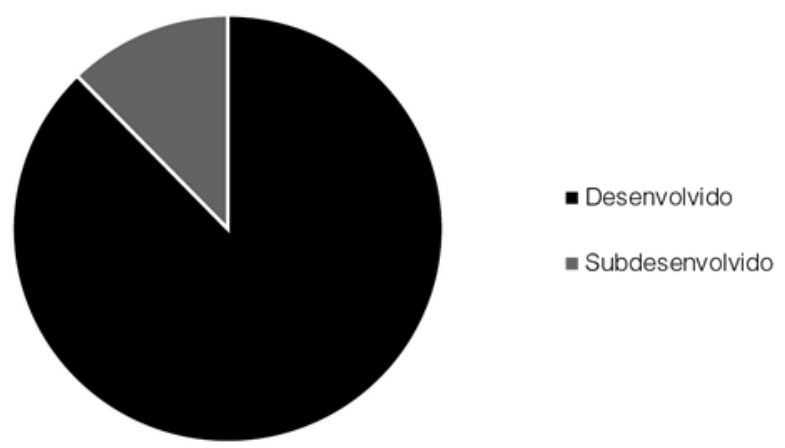

Fonte: Os autores.
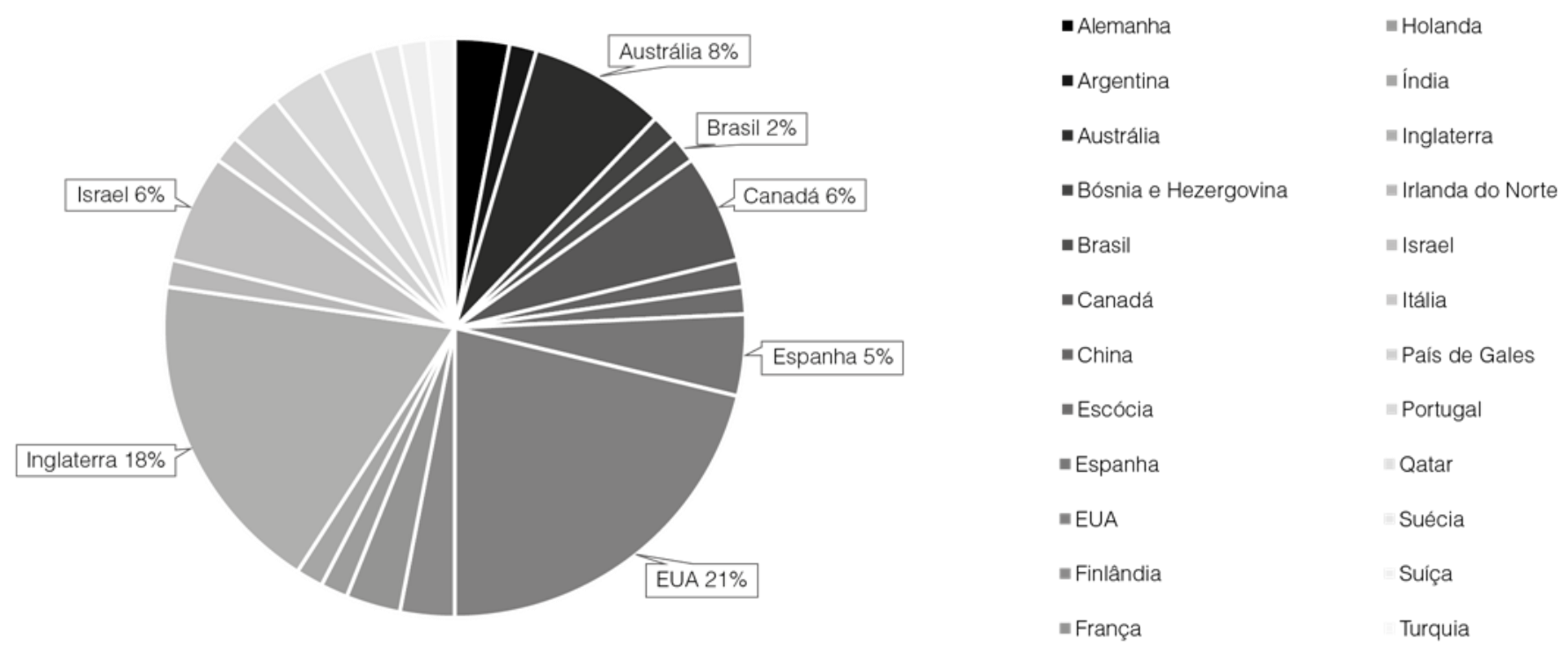

Fonte: Os autores. 
O Gráfico 3 distribui os artigos por ano de publicação, o que revela picos em 2013 e 2016

Gráfico 3 - Afiliação institucional do autor principal, por categoria de desenvolvimento

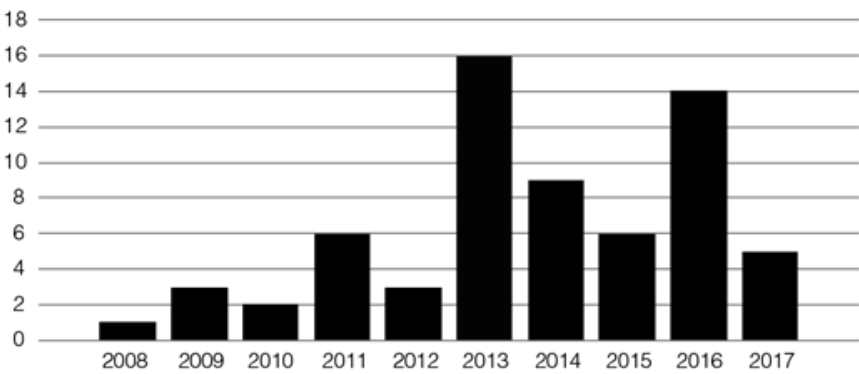

Fonte: Os autores.

O Gráfico 4 distribui a quantidade de vezes que cada obra de HL foi referenciada na amostra, sendo incluídas no gráfico apenas aquelas com mais de três ocorrências. Originais e traduções foram contados sob a mesma categoria, mas coletâneas foram consideradas como uma entrada própria. Destacam-se no gráfico, com contagens mais significativas: (1) A produção do espaço, referenciada em 53 dos 66 artigos da amostra; (2) $O$ direito à cidade, em 26; (3) Writings on cities, coletânea inglesa que reúne todos os textos de $O$ direito à cidade, parte daqueles de Espaço e política e Rhytmanalysis, e duas entrevistas, presente em 20 artigos; (4) e A revolução urbana, em 19. Note-se, portanto, que as referências a HL, mesmo entre aqueles que o estudam, são restritas a poucas obras. Nem mesmo todas as suas obras dedicadas ao urbano são recorrentes nas citações: Do rural ao urbano e Espaço e política são citados apenas três vezes cada um, e $O$ pensamento marxista e a cidade, quatro vezes.

\section{Discussão dos resultados}

Conforme anunciado na Metodologia (ver item 1), além da análise quantitativa (ver item 2), também foi realizada uma qualitativa, que procura complementar o método devido à especificidade do tema. Para isso, os textos foram examinados por meio de cinco questões, exibidas no Gráfico 5 e correspondentes às subseções a seguir: (3.1) trata de temas ligados ao subdesenvolvimento? (3.2) analisa experiências brasileiras? (3.3) possui objeto empírico? (3.4) apresenta renovação crítica da obra de HL? (3.5) relaciona forma social e forma urbana? (3.6) limites e possibilidades do método RSL segundo o objeto.

\section{$\mathrm{HL}+$ subdesenvolvimento}

O tema do subdesenvolvimento surge na pesquisa por causa de duas preocupações preliminares. A primeira, diz respeito à validade geral das teorias urbano-espaciais de HL: a explicação dos fenômenos urbanos e sociais agora sob a égide da globalização. Para tanto, procurou-se detectar também a abrangência geográfica dos estudos de caso abordados nos artigos, o que poderia indicar a utilização contemporânea dos conceitos e noções lefebvrianas para realidades outras que o contexto social original ao qual estavam em grande medida vinculados inicialmente. A segunda preocupação diz respeito à distribuição geográfica dos centros acadêmicos e instituições a que esses artigos estão afiliados. Ou seja: o quanto os países subdesenvolvidos são objetos e sujeitos da reflexão lefebvriana, considerados os critérios de escolha dos artigos, conforme o item 1.

Gráfico 4 - Quantidade de ocorrências na amostra para cada obra de Henri Lefebvre referenciada. Inserção apenas daquelas com mais de três ocorrências

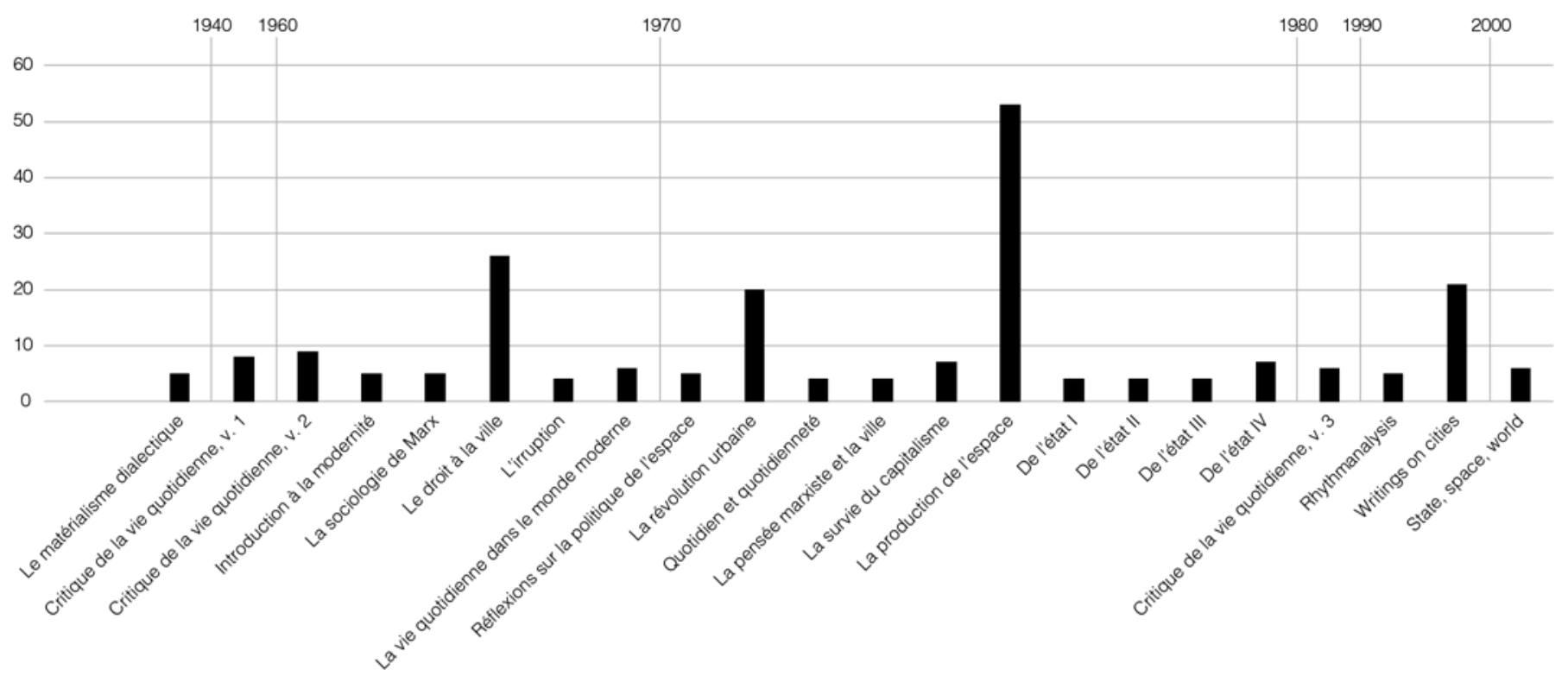

Fonte: Os autores. 
Gráfico 5 - Avaliação qualitativa da amostra

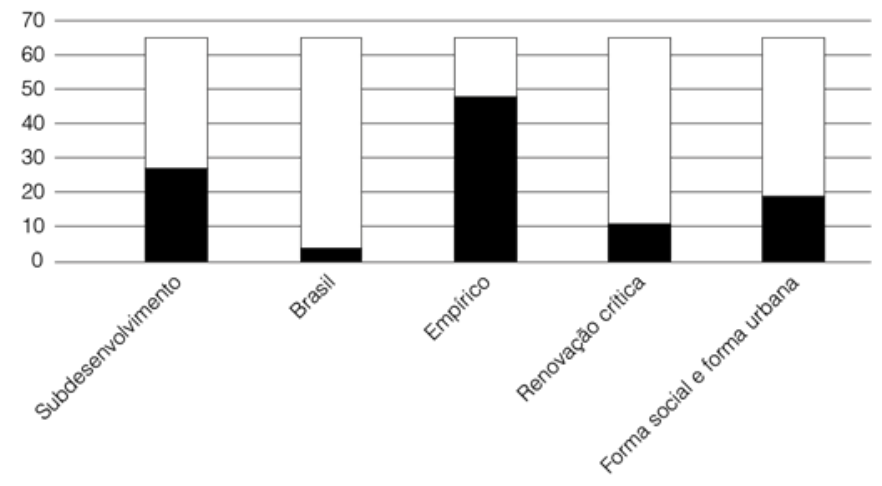

Fonte: Os autores

Um problema metodológico que se apresenta, portanto, é a separação geográfica segundo critérios de desenvolvimento. São diversas, e por vezes contraditórias, as abordagens possíveis. Por um lado, a separação mais consensual baseada em indicadores econômicos e sociais. Por outro, definições que consideram posições relativas ao sistema produtivo mundial - o Capitalismo. De modo mais incidente aos objetivos da pesquisa, vale indicar as noções de desenvolvimento e subdesenvolvimento mediadas por questões espaciais e sua reprodução desigual pelo globo. Para tanto, podemos nos valer da teoria do desenvolvimento geográfico desigual (SMITH, 2008), que permite aproximar as discussões no campo da economia sobre o desenvolvimento desigual e combinado para os aspectos mais diretamente ligados aos fenômenos espaciais em suas diferentes escalas.

Foram considerados como desenvolvidos os países integrantes da OCDE (Organização para Cooperação e Desenvolvimento Econômico) e com IDH (Índice de Desenvolvimento Humano) de estrato "muito alto" (superior a 0,8), conforme estabelecido pelo PNUD (Programa das Nações Unidas para o Desenvolvimento). Essa divisão inclui na categoria de países desenvolvidos

Gráfico 6 - Localização dos estudos de caso da amostra, por país

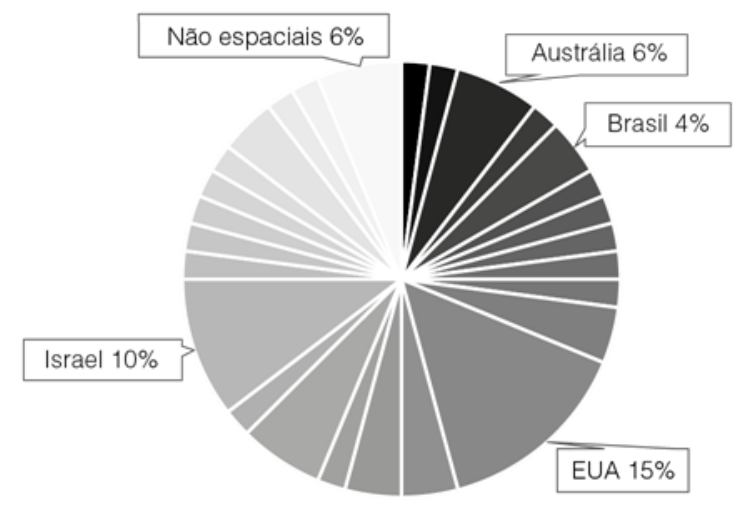

um grupo que deve ser relativizado, na medida em que, segundo outros critérios, não estaria arrolado entre os países centrais do capitalismo mundial. São esses: Canadá, Chile, Israel, Irlanda do Norte e Austrália. Considerou-se que essa discussão não alterava substancialmente o sentido dos objetivos buscados.

Dos 65 artigos analisados, 20 (30\%) tratam de temas teóricos, considerados de validade geral. A relevância desse grupo diz respeito ao fato de que faz parte da perspectiva lefebvriana a noção de "sociedade urbana", o que implica um processo "planetário" de urbanização (MERRIFIELD, 2013). Portanto, os objetos empíricos específicos desses artigos não existem, pelo menos no sentido geográfico aqui considerados.

Dentre os artigos analisados, apenas 15 (23\%) tratam de fenômenos espaciais de países subdesenvolvidos, e 30 (46\%), de realidades de países desenvolvidos (Gráficos 6 e 7). Da mesma maneira, 57 artigos (87\%) provêm de instituições de países desenvolvidos (Gráfico 2).

Esses dados indicam que, ainda que as teorias estudadas pretendam, e em grande medida alcancem a realidade global e planetária, as Instituições de Ensino Superior fora do eixo anglo-saxão têm pouca representação nos estudos lefebvrianos presentes na literatura indexada de excelência, marcadamente as situadas em países subdesenvolvidos. Ou seja, exatamente as realidades urbanas "radicais" que passaram por um processo de urbanização rápido e desordenado. Apenas um dos artigos da amostra tem afiliação brasileira, e trata de temas teóricos (SANTOS JUNIOR, 2014). Ainda, apenas quatro artigos tratam de questões relativas diretamente ao Brasil, produzidos por pesquisadores de instituições estrangeiras (BROWN, 2013; BELDA-MIQUEL; BLANES; FREDIANI, 2016; ÇELIK; GOUGH; 2014. KUYMULU, 2013).

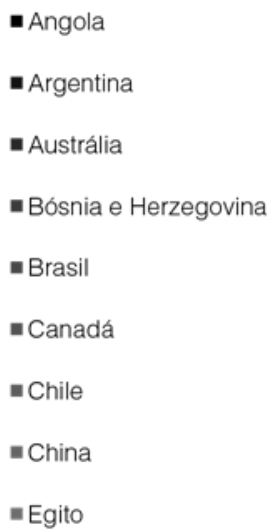

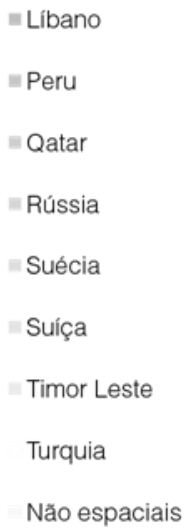

Fonte: Os autores 
A obra urbana de HL é muito presente no debate brasileiro, se considerarmos a precocidade da sua recepção na academia (ARANTES, 2009; MARTINS, 1996). Além desse fato, o mote "direito à cidade" (DC) consolidou-se tanto nas pautas reivindicatórias do movimento social quanto nas políticas públicas no início do século 21 . No entanto, a reflexão crítica que circula nos periódicos selecionados (ver item 2) não reflete essa realidade. $O$ Brasil não tem participado significativamente, nesses fóruns internacionais, nem como objeto de estudo nem como sujeito da reflexão.

Gráfico 7 - Localização dos estudos de caso da amostra, por categoria de desenvolvimento

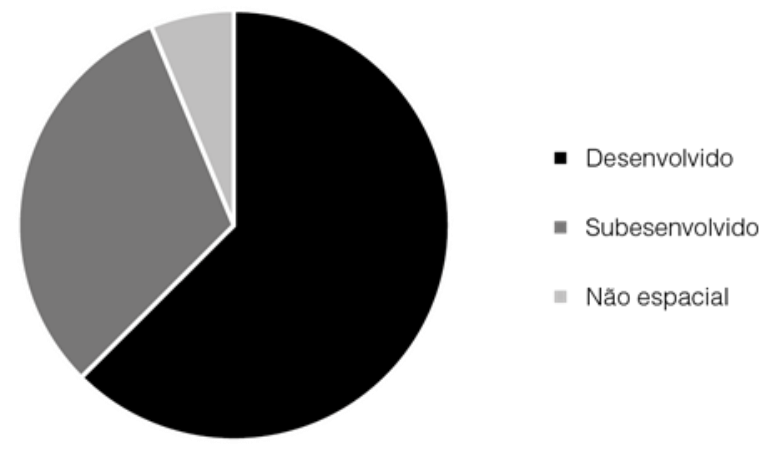

Fonte: Os autores.

$\mathrm{HL}+$ subdesenvolvimento + Brasil

Somente quatro artigos analisados tratam diretamente ou indiretamente do Brasil. Essa presença se dá segundo três principais questões: (1) as inovações jurídicoinstitucionais relativas à reforma urbana iniciadas com a Constituição de 1988 e consolidadas no Estatuto da Cidades de 2001 e com a criação do Ministério das Cidades em 2003; (2) as políticas públicas levadas a cabo segundo esse novo ordenamento jurídico pelas Prefeituras, Estados e Governo Federal; (3) os movimentos sociais pela reforma urbana, habitação e cidadania. Esse ciclo de experiências que caracterizou a redemocratização brasileira é a base sobre a qual os artigos se debruçam para analisar casos específicos. Todos os quatro artigos foram produzidos por pesquisadores e centro de pesquisas estrangeiros.

Os artigos indicados utilizam o caso brasileiro para poder pensar a institucionalização do DC, dado o caráter inovador da legislação e da governança a partir de 1988. A experiência brasileira - ter tornado lei o DC - permite uma plataforma a partir da qual os pesquisadores identificam avanços e problemas. Brown (2013) destaca positivamente o surgimento recente de uma agenda urbana na América Latina. No Brasil, especialmente, o autor aponta os avanços jurídicos e de governança realizados por meio dessa agenda como ferramenta contra a pobreza e a exclusão social.
Çelik e Gough (2014), em artigo que introduz a seção especial da revista Capital \& Class "Neoliberalismo urbano, estratégias para as lutas urbanas e o 'direito à cidade", destacam também, seguindo Neil Smith, os avanços legais e de políticas públicas brasileiros, em relação ao DC. Esse direito teria sido incorporado pelo processo brasileiro em contraposição à gentrificação, especialmente aquela promovida pelo Estado. Essa pauta foi, segundo a análise, trazida pelos movimentos sociais ligados ao Partido dos Trabalhadores, e inclui, especialmente, o orçamento participativo iniciado em Porto Alegre.

Kuymulu (2013) é crítico em relação à institucionalização do DC, apontando a perda de sua significação lefebvriana - criatividade e participação política. A institucionalização promovida sobreporia, segundo esse autor, a intermediação do "valor de troca" às outras possibilidades da ação política. Nesse sentido, o Fórum Mundial Social está mais próximo do verdadeiro DC que o V Fórum Urbano Mundial The right to the city: bridging the urban divide realizado no Rio de Janeiro. Neste último, a força dessa formulação lefebvriana se perde para a representação estatal e do big business diretamente envolvidos na produção do espaço urbano.

Nesse mesmo sentido crítico, Belda-Miquel, Blanes; e Frediani (2016) também analisam a incorporação institucionalizada, na conjuntura brasileira, do DC. No caso analisado, surge também o movimento social relativo às lutas urbanas, e a difícil relação, tomando o ponto de vista da discussão lefebvriana, entre os agentes sociais e o Estado. O exemplo do MSTB (Movimento dos Sem-Teto da Bahia) é ilustrativo da relação que conseguiu adquirir, nesse caso específico, independência política mesmo tendo obtido importantes vitórias e conquistas advindas do poder público. O MSTB teria demonstrado ser possível discernir o DC levado a cabo pelo Estado - e, portanto, sua institucionalização deletéria - dos objetivos políticos das populações em luta social. Diz:

Yet every time I see these 'machines for living in' I feel the $M S T B$ experience suggests an inspiring answer: the forces building the right to the city may demand that the state meet immediate needs, but should use these processes of struggle and the expectations created to promote social organization and self-management. (BELDA-MIQUEL; BLANES; FREDIANI, 2016, p. 336)

Ainda que HL seja um autor bastante divulgado entre nós, a relação de sua obra com temas arquitetônicos não tem sido discutida, no âmbito da literatura especializada. A profícua recepção política do DC não foi suficientemente dialetizada com a dimensão espacial, considerada como "prático-sensível", e fundamental para o autor. Tal dimensão implica tanto a arquitetura e os edifícios quanto a forma urbana, em constante relação. É só considerando 
essa mútua determinação entre forma e conteúdo que pode ser entendida a sua crítica à modernidade urbanoarquitetônica.

Nesse sentido, a reflexão lefebvriana que envolve diretamente o espaço das cidades é fundamental para o contexto brasileiro, na medida em que unifica a crítica estética e social do espaço moderno, entendido como "ideologia" e contrário à "totalidade aberta" que é a cidade. Seguindo suas formulações, podemos dar corpo à ideia da anti-urbanidade estruturante da forma social, urbana e arquitetônica brasileiras. Não apenas as dificuldades de "participação" nos processos de produção do espaço urbano, mas principalmente as estratégias de segregação de nossos paradigmas espaciais, interferem historicamente em nossas decisões arquitetônicas.

\section{$H L+$ objetos empíricos}

Dentre os artigos analisados, $73 \%$ (48 de 65) utilizam estudos de casos como parte de sua metodologia, sendo que 62\% (30 de 48) desses estudos de casos estão localizados em países desenvolvidos, $31 \%$ (15 de 48) em países subdesenvolvidos, e $6 \%$ (3 de 48) não têm objetos espaciais (ver Gráficos 5 e 6). Destacam-se, nessa amostragem, os temas relacionados a áreas urbanas em processo de transformação urbana ou urbanização (e.g. BALZARINI; SHLAY, 2016; DIXON, 2013; OAKLEY, 2014).

Nota-se que, quando os estudos de casos são parte da metodologia, os temas lefebvrianos mais recorrentes são os que referem-se ao DC como direito de "participação" e/ou utilização comunitária, nos termos do urbanismo bottom-up (e.g. BOWER, 2016; PIERCE; WILLIAMS; MARTIN, 2016). Também se destacam as analogias aos conceitos de "espaço vivido, percebido e concebido", presentes na obra A produção do espaço, de 1974. Esses conceitos são utilizados tanto para comprovar a pertinência de alguns processos urbanos bem-sucedidos, quanto para revelar os problemas das intervenções vinculadas às práticas predatórias do capitalismo neoliberal, que acentuam a distância entre a ideia de uma "cidade-obra" e os sistemas urbanos vinculados aos interesses do capital privado. Ademais, os artigos convergem no que diz respeito à relação dos seus objetivos e a metodologia adotada, ou seja, utilizam-se de exemplos práticos para comprovar a relevância e atualidade dos principais conceitos decorrentes da obra de HL.

Como exemplo, o artigo "Disneyfication and localisation: the cultural globalisation process of Hong Kong Disneyland" (CHOI, 2012), que trata o caso da Disneylândia de Hong Kong, fundada em 2005. Por meio da análise deste megaempreendimento global os autores destacam seu fracasso econômico como reflexo da desterritorialização de certas práticas locais - em descompasso com o rígido sistema impetrado pela matriz da Disneylândia. Por outro lado, alguns dos artigos analisados (e.g. AĞLARGÖZ, 2017; BALZARINI; SHLAY, 2016; FRIEDMAN, 2010) ressaltam que esse tipo de participação pode ocultar interesses particulares de grupos urbanos (moradores, mercado, comerciantes) por detrás de reivindicações "comunitárias" - aparentemente destinadas ao bem comum de todos os envolvidos. Nesse sentido, esta pesquisa destaca a relevância do aperfeiçoamento das metodologias de participação, com atenção à qualidade das informações disponibilizadas, à especificação clara do que compete ao corpo técnico e à transparência no processo de ideação e execução. Caso contrário, as práticas predatórias do mercado imobiliário podem ser intensificadas e legitimadas por mecanismos presumidamente democráticos.

Outro exemplo, o artigo “The Transparency of Democracy': The Production of Washington's Nationals Park as a Late Capitalist Space" (FRIEDMAN, 2010), ressalta as estratégias de manipulação do capital em relação ao que aos conceitos de "espaço vivido, percebido e concebido". Segundo os autores, os Nationals Parks de Washington utilizam-se de instrumentos vinculados aos espaços concebidos que atuariam nos espaços percebido e vivido, como forma de criar uma ilusão de cidade transparente e democrática, pois é parte estratégica do processo a marginalização da população mais pobre.

De modo geral, a RSL aqui apresentada revela que em muitos dos artigos que trabalham com estudos de casos como instrumento metodológicos, os conceitos lefebvrianos aparecem de forma equivocada ou excessivamente simplificada. Como, por exemplo, a relação entre DC e a participação da população nas políticas urbanas ou mesmo "a produção do espaço" como tema exclusivo dos mecanismos de mercado. Destarte, a diversificação das metodologias de análise da obra de HL torna-se instrumento essencial à atualização desse autor à complexa conjuntura social, territorial e política do século 21.

\section{$\mathrm{HL}$ + aproximações teóricas + renovação crítico- teórica}

Consideramos como "aproximações teóricas" os artigos que discutem diretamente conceitos, noções e princípios elaborados por HL em sua obra urbano-espacial. Encontramos com essa classificação um total de 20 artigos (30\%). Esse item indica a preocupação em relação à continuidade do pensamento crítico lefebvriano, que deveria acompanhar as transformações sociais das últimas décadas. Seu pensamento define categorias com validade abrangente, na medida em que possibilita, de forma original, a compreensão espacial de dinâmicas sociais. Talvez seja esse o principal motivo de sua intensa utilização explicativa de casos específicos, e que não se 
ocupam diretamente da atualização desse pensamento que, em um primeiro momento, tem grande potencialidade para descrever e criticar situações socioespaciais. Essa discussão, na maioria dos casos, prescinde da necessidade da atualização teórica desse pensamento.

A maioria dos artigos analisados (70\%) identifica o potencial do arsenal teórico de HL para explicar fenômenos distintos, o que também pode indicar sua atualidade. Porém, essa utilização não resulta em discussões teóricas e metodológicas mais abrangentes, já que seu interesse principal é o estudo de casos e eventuais estratégias de enfrentamento de problemas detectados.

Os artigos considerados teóricos, no entanto, refletem a urgência em discutir as repercussões conceituais decorrentes das drásticas alterações espaciais contemporâneas, bem como o grau crescente de alienação da "vida cotidiana". Essa atualização implica identificar e criticar pontos cegos dessa teoria e alterar noções em função do processo social. O caminho apontado pela maioria dos trabalhos considerados é, no entanto, a confirmação de sua validade teórica e sua utilidade na compreensão de novos fatos.

Dentre os artigos considerados como teóricos, apenas 11 dos artigos analisados (16\%) procuram um diálogo conceitual com o autor, expandindo e renovando suas ideias, criticando-os ou propondo novas abordagens para além de suas noções originais. Em relação aos autores que procuram desdobramentos do corpus teórico em direção a temas emergentes nos últimos anos, ou ainda, novas ênfases ditadas pelo debate contemporâneo, podemos identificar: a questão da autogestão discutida segundo a participação (BOWER, 2016); a problemática da colonização (KIPFER; GOONEWARDENA, 2013); a discussão sobre espaço e as novas tecnologias (READ; DE LAAT-LUKKASSEN; JONAUSKIS, 2013); além do DC confrontado a novas formas de luta social, como o Occupy (MERRIFIELD, 2013) e a institucionalização dos movimentos sociais e do DC (BELDA-MIQUEL; BLANES; FREDIANI, 2016), que exigem uma nova "virada" crítica; reafirmar o caráter crítico do pensamento de HL é também a intenção de Kipfer, Saberi e Wieditz (2012), contra sua utilização instrumental corrente.

Destaca-se, nesse sentido, a discussão sobre o prolongamento contemporâneo da noção lefebvriana de "cidade-obra" em direção à nova cidadania e ao desenhourbano (CHIODELLI, 2013). A dimensão formal da cidade ("prático-sensível") teria sido subvalorizada na recepção da obra de HL, quando, segundo o artigo, é um de suas contribuições mais importantes. Já Kipfer e Goonewardena (2013) procuram explorar o problema da colonização como dominação que não se encerra com a descolonização formal. Os processos que envolvem a dominação colonial prolongam-se por meio de diferentes relações socioespaciais, ocupando consciências e se expressando em dinâmicas culturais. Apesar de sua obra estar centrada na discussão sobre a vida cotidiana, o artigo aponta que HL não teria explorado as formas particulares de alienação que resultam desse processo, indicando assim a necessidade de desdobramento de seu pensamento em função de alterações sociais recentes.

A utilização instrumental das reflexões lefebvrianas é um dos principais problemas dessa teoria hoje. Seu potencial de análise e sua abrangência incomum têm permitido sua utilização como instrumento tanto para a compreensão quanto para resolução de problemas genéricos, os quais não seriam provavelmente objeto dessa teoria socialmente crítica e rigorosa. No universo dos artigos analisados, o exemplo mais acentuado dessa utilização tópica é o caso da aplicação da tríade espacial lefebvriana (pensado, vivido e percebido) para fundamentar uma nova "estética corporativa" que procura entender os espaços das grandes empresas ou órgãos governamentais a fim de resolver, sem rupturas, problemas de identidade cultural e de gênero (WASSERMAN; FRENKEL, 2015). A utilização instrumental mais grave, no entanto, teria ocorrido com o próprio DC. Quanto a esse risco, Merrifield (2011) já detectava a preocupação do próprio $\mathrm{HL}$ em um de seus últimos textos (LEFEBVRE, 1989). Segundo ele, HL "insiste que, nesse caso, se deve abandonar o conceito [que não mais funciona], presenteá-lo ao inimigo" (MERRIFIELD, 2011, p. 109). Assim HL compreendera $o$ processo de institucionalização - tecnocracia e burocracia - do DC, e buscava saída para retomar o seu caráter revolucionário e novo.

\section{$\mathrm{HL}+$ forma social e forma urbana}

Mesmo com os critérios de busca adotados pela seleção, do total de 64 artigos, apenas 19 (29\%) de modo mais direto trata das dimensões "prático-sensíveis" do urbano. A maioria (70\%) enfrenta de modo indireto essa questão, sendo que nenhum deles aborda diretamente o Brasil como caso de estudo. Esse resultado corrobora uma das principais hipóteses por nós aventadas - a de que, no contexto nacional, a relação dos conceitos lefebvrianos utilizados após o crash de 2008 vincula-se sobretudo às formas de gestão ou ao ativismo bottom-up como instrumentos de combate fundamentais à alienação urbana e social advinda do ciclo neoliberal iniciado nos anos 1980.

Contudo, as pesquisas e seminários realizados pelo [oculto para anonimato], têm revelado que a obra de HL ressalta em diversos momentos a complexidade das relações entre a forma urbana - seu conteúdo prático sensível - e a forma social. Ou seja, HL considera que a relação entre as externalidades das decisões espaciais conferidas em determinadas soluções da ordem do urbanismo ou mesmo da arquitetura, conferem uma relação dialética com os 
conteúdos sociais de um determinado local. Como por exemplo:

\begin{abstract}
Whenever I step foot in Mourenx I am filled with dread. Yet the new town has a lot going for it. The overall plan (the master blueprint) has a certain attractiveness: the lines of the tower blocks alternate horizontals and verticals. The break between the landscape - wooded hills, moorland, vineyards - and the city may be rather abrupt, but it is bearable; it is relatively easy on the eye. The blocks of flats look well planned and properly built [...]
\end{abstract}

Yet every time I see these 'machines for living in' I feel terrified. [...] These blocks of flats are also 'technological objects' and machines. Will they be able to provide a new humanism? Are they already providing it? Can they mediate between man and nature, between one man and another? Are they bringing individuals, families and groups together, or are they forcing them apart? [...] Are we entering the city of joy or the world of unredeemable boredom? (LEFEBVRE, [1962] 1995, p. 118-119)

$A$ vida urbana, a sociedade urbana, numa palavra 'o urbano' não podem dispensar uma base prático-sensivel, uma morfologia. (LEFEBVRE, [1968] 2008, p. 49)

Quando a relação entre forma urbana e forma social se dá nas condições territoriais dos países subdesenvolvidos, como no caso do Brasil, sua operacionalidade é amplificada, visto que a desigualdade econômica acentua as diferenças espaciais. Destarte, se considerarmos a arquitetura e o urbanismo como problemas do urbano, ou melhor das cidades, a compreensão disciplinar em ambiente desigual e combinado requer estratégias teóricas específicas, para que os objetos empíricos tratados possam ser analisados em relação à tensão estabelecida com a cidade-obra.

$\mathrm{HL}+\mathrm{RSL}$

Em relação a nosso tema, o método RSL proposto nessa edição especial da revista PARC permitiu a compreensão tanto de seus limites quanto possibilidades. No que diz respeito aos limites, verificou-se que a necessidade de uma leitura qualitativa essencial ao conteúdo lefebvriano esbarra na grande disseminação da obra urbano-espacial desse autor. Por isso, optou-se por reduzir a amostragem conforme as indicações na seção "Metodologia" (item 1). Quanto a suas possibilidades, a sistematização da pesquisa bibliográfica detectou importantes conexões que não seriam possíveis de outra forma. Ela revelou uma geografia de autores, talvez ignoráveis em um levantamento assistemático, bem como evidenciou uma utilização instrumental do arcabouço conceitual lefebvriano bastante problemático.

\section{Considerações finais}

Mesmo considerando os limites e as especificidades dos critérios de filtragem que buscaram a relação entre a obra de HL sobre o espaço e a cidade e as preocupações mais diretamente voltadas para a arquitetura e desenho urbano, apenas uma menor parte dos artigos trata disso diretamente (ou seja, a recepção dessa obra dentro de um campo disciplinar específico). Tendo como base a aproximação proposta e os critérios de busca, conclui-se que a influência da obra lefebvriana nos temas arquitetônicos é pouco expressiva, pelo menos no universo das publicações acadêmicas alcançadas. Isso é um problema, se considerarmos as possibilidades compreensivas e críticas dessa obra. Deve-se ressaltar, no entanto, que a obra de HL é uma referência decisiva no debate acadêmico das últimas décadas, o que coloca sua influência para além da citação direta, na qual se baseia a proposta metodológica deste artigo. A perspectiva de análise proposta se refere sobretudo à aplicação de HL de um ponto de vista disciplinar, e não à obra enquanto tal.

$\mathrm{O}$ ainda pequeno número de publicações relativas à realidade brasileira ou subdesenvolvida, nesse universo pesquisado, também é destaque. Tanto mais importante se considerada a urgência da questão urbana e espacial nessas geografias, principalmente sob as determinações da globalização e a mais recente apropriação imediatamente política do DC.

\section{Agradecimentos}

Agradecemos à Fapesp (Processos 2015/19847-9 e 2016/21407-0) e CNPq (Bolsa Produtividade).

\section{Referências}

AĞLARGÖZ, O. "We are at this campus, there is nothing in this campus ...": Socio-spatial analysis of a university campus.

Tertiary Education and Management, v. 23, n. 1, p. 69-83, 2 jan. 2017. DOI: https://dx.doi.org/10.1080/13583883.2016.1207798

ARANTES, O. B. F. Urbanismo em fim de linha: e outros estudos sobre o colapso da modernização arquitetônica. São Paulo: Edusp, 1998. 224 p.

ARANTES, P. F. Em busca do urbano: marxistas e a cidade de São Paulo nos anos de 1970. Novos Estudos-CEBRAP, n. 83, p. 103-127, 2009. DOI: https://dx.doi.org/10.1590/S0101-33002009000100007 
BALZARINI, J. E.; SHLAY, A. B. Gentrification and the Right to the City: Community Conflict and Casinos. Journal of Urban Affairs, v. 38, n. 4, p. 503-517, out. 2016. DOI: http://dx.doi.org/10.1111/juaf.12226

BELDA-MIQUEL, S.; BLANES, J. P.; FREDIANI, A. Institutionalization and Depoliticization of the Right to the City: Changing Scenarios for Radical Social Movements. International Journal of Urban and Regional Research, v. 40, n. 2, p. 321-339, mar. 2016. DOI: http://dx.doi.org/10.1111/1468-2427.12382

BOWER, R. Who Decides and Who Provides? The Anarchistic Housing Practices of John Turner as Realizations of Henri Lefebvre's Autogestive Space. Alternatives: Global, Local, Political, v. 41, n. 2, p. 83-97, maio 2016. DOI: https://doi.org/10.1177/0304375416684856

BROWN, A. The right to the city: road to Rio 2010. International Journal of Urban and Regional Research, v. 37, n. 3, p. 957971, maio 2013. DOI: https://dx.doi.org/10.1111/1468-2427.12051

ÇELIK, Ö.; GOUGH, J. Introduction to the special section: Urban neoliberalism, strategies for urban struggles, and "the right to the city”. Capital \& Class, v. 38, n. 2, p. 414-451, 2014. DOI: https://dx.doi.org/10.1177/030981681453329

CHIODELLI, F. Planning and urban citizenship: suggestions from the thoughts of Henri Lefebvre. Planning Perspectives, v. 28, p. 487-494, 2013. DOI: http://dx.doi.org/10.1080/02665433.2013.800717

CHOI, K. Disneyfication and localisation: the cultural globalisation process of Hong Kong Disneyland. Urban Studies, v. 49, n. 2, p. 383-397, fev. 2012. DOI: https://dx.doi.org/10.1177/0042098011402234

DIXON, M. Transformations of the spatial hegemony of the courtyard in post-soviet St. Petersburg. Urban Geography, v. 34, n. 3, p. 353-375, maio 2013. DOI: http://dx.doi.org/10.1080/02723638.2013.778663

DRESCH, A.; LACERDA, D. P.; ANTUNES JR., J. A. V. Design science research: a method for science and technology advancement. Heidelberg, Nova York, Dordrecht, Londres: Springer, 2015. 163 p.

FRIEDMAN, M. T. "The transparency of democracy": the production of Washington's nationals park as a late capitalist space. Sociology of Sport Journal, v. 27, n. 4, p. 327-350, 2010.

HARVEY, D. Rebel cities: from the right to the city to the urban revolution. Londres, Nova York: Verso, 2012.208 p.

JAMESON, F. A cultura do dinheiro: ensaios sobre a globalização. 2ª ed. Petrópolis: Vozes, 2002. 207 p.

KIPFER, S.; SABERI, P.; WIEDITZ, T. Henri Lefebvre: debates and controversies. Progress in Human Geography, v. 37, n. 1, p. 115-134, 2012. DOI: https://dx.doi.org/10.1177/0309132512446718

KIPFER, S.; GOONEWARDENA, K. Urban marxism and the post-colonial question: Henri Lefebvre and "colonisation".

Historical Materialism, v. 2, n. 21, p. 76-116, 2013. DOI: https://dx.doi.org/10.1163/1569206X-12341297

KOOLHAAS, R.; MAU, B. S, M, L, XL. New York: The Monacelli Press, 1995. 1344 p.

KUYMULU, M. B. The vortex of rights: "right to the city" at a crossroads. International Journal of Urban and Regional Research, v. 37, n. 3, p. 923-940, maio 2013. DOI: https://dx.doi.org/10.1111/1468-2427.12008

LEFEBVRE, H. Quand la ville se perd dans une métamorphose planétaire. Le monde diplomatique, p. 16-17, maio 1989.

LEFEBVRE, H. Introduction to modernity: twelve preludes. 3ª ed. Londres, Nova York: Verso, 1995. 402 p.

LEFEBVRE, H. O direito à cidade. 5a. ed. São Paulo: Centauro, 2008. 143 p.

LEFEBVRE, H. Toward an Architecture of Enjoyment. Tradução Robert Bononno. Minneapolis: University of Minnesota Press, 2014. $190 \mathrm{p}$.

MARTINS, J. de S. Henri Lefebvre e o retorno à dialética. São Paulo: Hucitec, 1996. 151 p. 
MERRIFIELD, A. El derecho a la ciudad y más allá: notas sobre una reconceptualización lefebvriana. Urban, v. 3, p. 101-110, 2011.

MERRIFIELD, A. The urban question under planetary urbanization. International Journal of Urban and Regional Research, v. 37, n. 3, p. 909-922, maio 2013. DOI: https://dx.doi.org/10.1111/j.1468-2427.2012.01189.x

OAKLEY, S. A lefebvrian analysis of redeveloping derelict urban docklands for high-density consumption living, Australia. Housing Studies, v. 29, n. 2, p. 235-250, 17 fev. 2014. DOI: http://dx.doi.org/10.1080/02673037.2014.851175

PIERCE, J.; WILLIAMS, O. R.; MARTIN, D. G. Rights in places: An analytical extension of the right to the city. Geoforum, v. 70, p. 79-88, mar. 2016. DOI: http://dx.doi.org/10.1016/j.geoforum.2016.02.006

READ, S.; DE LAAT-LUKKASSEN, M.; JONAUSKIS, T. Revisiting “complexification," technology, and urban form in Lefebvre. Space and Culture, v. 16, n. 3, p. 381-396, 2013. DOI: https://dx.doi.org/10.1177/1206331213487060

SANTOS JUNIOR, O. A. DOS. Urban common space, heterotopia and the right to the city: Reflections on the ideas of Henri Lefebvre and David Harvey. URBE - Revista Brasileira de Gestão Urbana, v. 6, n. 541, p. 146, 2014. DOI: https://dx.doi.org/10.7213/urbe.06.002.SE02

SMITH, N. Uneven development: nature, capital, and the production of space. $3^{\mathrm{a}}$ ed. Atenas: University of Georgia Press, 2008. $323 \mathrm{p}$.

STANEK, L. Henri Lefebvre on space: architecture, urban research and the production of theory. Minneapolis: University of Minnesota Press, 2011.371 p.

STANEK, Ł.; SCHMID, C.; MORAVÁNSZKY, Á. Urban revolution now: Henri Lefebvre in social research and architecture. Burlington: Ashgate Pub, 2014. 345 p.

WASSERMAN, V.; FRENKEL, M. Spatial work in between glass ceilings and glass walls: gender-class intersectionality and organizational aesthetics. Organization Studies, v. 36, n. 11, p. 1485-1505, 2015. DOI:

https://dx.doi.org/10.1177/0170840615593583

\section{${ }^{1}$ Leandro Silva Medrano}

Arquiteto e Urbanista. Livre-docente. Rua do Lago, 876, Butantã, São Paulo, SP, Brasil, 03178-200

\section{${ }^{2}$ Luiz Antonio Recamán Barros}

Arquiteto e Urbanista, Cientista Social. Doutor. Rua do Lago, 876, Butantã, São Paulo, SP, Brasil, 03178-200

\section{${ }^{3}$ Mariana Martinez Wilderom Chagas}

Arquiteta e Urbanista. Mestre, Doutoranda pelo Programa de Pós-Graduação em Arquitetura e Urbanismo da FAUUSP. Rua do Lago, 876, Butantã, São Paulo, SP, Brasil, 03178-200

\section{${ }^{4}$ Raphael Grazziano}

Arquiteto e Urbanista. Doutorando pelo Programa de Pós-Graduação em Arquitetura e Urbanismo da FAUUSP. Rua do Lago, 876, Butantã, São Paulo, SP, Brasil, 03178-200 